\title{
Revista
}

\section{DIREITOS HUMANOS}

fundamentais

\section{edifieo}


Editor Responsável Luiz Fernando da Costa e Silva

Coordenaçáo Editorial Luís Rodolfo Ararigboia de Souza Dantas

Comissáo Editorial Ana Maria de Pinho

Projeto Gráfico Carla de Araújo Risso

Técnico Editorial / Diagramaçáo / Capa Rodolfo Rodrigues Domingos

Direitos reservados à Campus Vila Yara

EDIFIEO Editora da FIEO Av. Franz Voegeli, 300 Bloco Branco

06020-190 Osasco SP Brasil

Fone 1136519980

www.unifieo.br

edifieo@unifieo.br

Ficha Catalográfica elaborada pela

Biblioteca Prof. Dr. Luiz Carlos de Azevedo

Revista Direitos Humanos Fundamentais / UNIFIEO - Centro

Universitário FIEO. Programa de Pós-Graduação em Direito.

Stricto sensu. Ano 16, n.2 (2016) - Osasco : EDIFIEO, 2016.

v. ; $26 \mathrm{~cm}$

Semestral

Continuação de: Revista Mestrado em Direito. Direitos

Humanos Fundamentais.

ISSN: 2358-9949

1. Direitos Humanos - Periódicos. I. UNIFIEO - Centro Universitário FIEO. Programa de Pós-Graduação em Direito.

Stricto sensu.

Campus Vila Yara

Av. Franz Voegeli, 300 06020-190 Osasco SP Brasil
Campus Wilson

Av. Franz Voegeli, 1743

06020-190 Osasco SP Brasil 


$\begin{aligned} \text { Reitor e Pró-Reitor Administrativo } & \text { Edmo Alves Menini } \\ \text { Pró-Reitor Acadêmico } & \text { Nivaldo Elias Pilão } \\ \text { Pró-Reitor de Extensáo e Cultura } & \text { Fernando Pavan Baptista } \\ \text { Pró-Reitor de Desenvolvimento e } & \\ \text { Relaçóes Comunitárias } & \text { Antonio Claudio da Costa Machado }\end{aligned}$

Coordenadora do Curso de Mestrado Anna Candida da Cunha Ferraz

Editor Chefe Luís Rodolfo Ararigboia de Souza Dantas

Editora Adjunta Anna Candida da Cunha Ferraz

Editor Adjunto Gerson Amauri Calgaro

Editora Adjunta Margareth Anne Leister

Revisor Antonio Cláudio da Costa Machado

Revisora Ana Maria de Pinho 


\section{Conselho Editorial}

\section{Conselheiros Nacionais}

Alexandre de Moraes - USP/FD/SP

Arnaldo Sampaio de Moraes Godoy - UNICEUB/ $\mathrm{FD} / \mathrm{DF}$

Carlos Luiz Strapazzon - UNOESC/FD/SC

Eduardo Biacchi Gomes - UNIBRASIL/FD/PR

Eduardo Carlos Bianca Bittar - USP/FD/SP

Elival da Silva Ramos - USP/FD/SP

Eneá de Stutz e Almeida - UNB/FD/DF

Fernanda Dias de Menezes - USP/FD/SP

Georgenor de S. Franco Filho - UNAMA/FD/PA

Gustavo Just - UFPE/FD/PE

Ingo Wolfgang Sarlet - PUC/FD/RS

João Maurício Leitão Adeodato - FDV/ES

José Bittencourt Filho - UNB/FD/DF

Joyceane Bezerra de Menezes - UNIFOR/FD/CE

Luciana Aboim Machado Gonçalves da Silva - UFS/ $\mathrm{FD} / \mathrm{SE}$

Livia Gaigher Bosio Campello - UFMS/FD/MS;

Maria Garcia - PUC/FD/SP

Maria Luiza P. de Alencar Mayer Feitosa - UFPB/ $\mathrm{FD} / \mathrm{PB}$

Sérgio de Andréa Ferreira - UERJ/FD/RJ

Susana Camargo Vieira - UNIVERSIDADE DE ITAÚNA/FD/MG

Valério Oliveira Mazzuoli - UFMT /FD/ MT

\section{Conselheiros Estrangeiros}

Alberto Levi - Universidade de Modena e Reggio Emília, FD/MILĀO/ITÁLIA

Alejandro Miguel Garro - Universidade de Columbia/FD/NEW YORK/ USA

Andrea Romano - Università di Roma "La Sapienza"/FD/ITÁLIA

Angelo Schillaci - Università degli studi di Teramo/ FD/ITÁLIA

Arturo Martinez y Gonzalez - Universidade Autônoma/FD/MÉXICO

Diogo Leite de Campos - Universidade de Coimbra/ FD/PORTUGAL

Francisco Fernández Segado - Universidad Complutense de Madrid/FD/ESPANHA

Holger Knudsen - Max Plancker Instit fuer auslaendisches um internationales Privatrecht/ HAMBURGO/ALEMANHA

Hugo Mansueti - Fac. Católica de Buenos Aires / FD/ARGENTINA

João Leal Amado - Universidade de Coimbra/FD/ PORTUGAL

Jorge Bacelar Gouveia - Universidade Nova de Lisboa/FD/PORTUGAL

Jorge Miranda - Universidade de Lisboa/FD/ PORTUGAL

Nuno Piçarra - Universidade Nova Lisboa/FD/ PORTUGAL

Teodósio Palomino Ramirez - Universidade Tecnológica do Peru/FD/PERU

Ursula Cristina Basset - Pontificia Universidad Católica Argentina /FD/ARGENTINA

Victor Bázan - Universidade Católica de Cuyo/FD/ San Juan/ARGENTINA 


\section{Nominata de Conselheiros e \\ Pareceristas ad hoc desta edição}

\section{Nominata de Pareceristas}

Carlos Alberto Molinaro - PUC/FD/RS

Arnaldo Sampaio de Moraes Godoy - UNICEUB/ $\mathrm{FD} / \mathrm{DF}$

Robison Tramontina - UNOESC/FD/SC

Márcio Ricardo Staffen - IMED/FD/RS

Laura Souza Lima e Brito - UFMG/FD/MG

Laura Garbini Both - UNIBRASIL/FD/ PR

Ivelise Fonseca da Cruz - FMU/FD/SP

Ursula Cristina Basset - Pontificia Universidad Católica Argentina /FD/ARGENTINA

Valéria Ribas do Nascimento - UFSN/FD/RS

Maria Cristina Pezzela - UNOESC/FD/SC

Rogerio Luiz Nery da Silva - UNOESC/FD/SC

Rosa Aguilar Verástegui -UEL/FD/PR

Carlos Ugo Santander - UFG/FD/GO

Marcia Cristina de Souza Alvim - PUC/FD/SP

Heloisa Fernandes Câmara - UFPR/FD/PR

Tomás Filipe Schoeller Paiva - USP/FD/SP

Carlos Luiz Strapazzon - UNOESC/FD/SC

Leonardo da Rocha de Souza - FURB/FD/SC

Fernando Joaquim Ferreira Maia - UFRPE/FD/PE

Marília de Nardin Budó - IMED/FD/RS 



\section{SUMÁR10}

Editorial 11

\section{DOUTRINA ESTRANGEIRA}

O "Sistema Europeu Comum de Asilo" à prova da "crise migratória e de refugiados sem precedentes"

\section{Nuno Picarra}

\section{DOUTRINA NACIONAL}

Contribuições de Isaiah Berlin para refletir sobre liberdade

Jan Gustave de Souza Havlik

Gabriela Maia Rebouças

A prática Jurídica Social no curso de Direito da Universidade Federal do Rio Grande, Brasil

\section{Renato Duro Dias}

O aborto: um olhar sob a perspectiva dos Direitos à Privacidade e à Autonomia Decisória

Riva Sobrado de Freitas

O Direito Fundamental de acesso à informação pública e a importância da transparência ativa

\section{Marcelo Rodrigues Mazzei \\ Marcelo de Senzi Carvalho \\ Zaiden Geraige Neto}

O papel da família na educação para o uso da internet: proteção à criança e ao adolescente

\section{Mirian Andrade Santos}

O papel do conselho federal da Ordem dos Advogados do Brasil no controle de constitucionalidade de Direitos Fundamentais 
Restrições aos Direitos Fundamentais

Andre Luiz dos Santos Nakamura

O valor constitucional dos protestos populares - o fim dos "inocentes do Leblon" 167

Dimitri Dimoulis

Soraya Regina Gasparetto Lunardi

Normas para publicação 179 


\section{FOREIGN AUTHORS}

The "Common European Asylum System" to proof of the "unprecedented migratory and refugee crisis".

\section{Nuno Picarra}

\section{NATIONAL AUTHORS}

Isaiah Berlin's contributions to reflect on freedom .47

Jan Gustave de Souza Havlik

Gabriela Maia Rebouças

Social legal practice in law course at the Universidade Federal do Rio Grande, Brazil

\section{Renato Duro Dias}

Abortion: a look under the perspective of rights to privacy and decisional autonomy

\section{Riva Sobrado de Freitas}

The Fundamental Rights of access to public information and the importance of the active transparency

\section{Marcelo Rodrigues Mazzei \\ Marcelo de Senzi Carvalho \\ Zaiden Geraige Neto}

The role of the family in education for internet use: protection of children and adolescents

\section{Mirian Andrade Santos}

The role of the brazilian bar association in the judicial review in the cases about Fundamental Rights 
Restrictions of Fundamental Rights

Andre Luiz dos Santos Nakamura

The constitutional value of popular protests - the end of the "innocents of

Leblon" 167

Dimitri Dimoulis

Soraya Regina Gasparetto Lunardi

Instructions for publication 179 